\title{
Silencing of Annexin A1 suppressed the apoptosis and inflammatory response of preeclampsia rat trophoblasts
}

\author{
JING FENG, XINLING WANG, HONGYAN LI, LI WANG and ZENGJUN TANG \\ Department of Gynaecology and Obstetrics, Hebei General Hospital, Shijiazhuang, Hebei 050051, P.R. China
}

Received May 2, 2018; Accepted September 11, 2018

DOI: $10.3892 / \mathrm{ijmm} .2018 .3887$

\begin{abstract}
Preeclampsia (PE) is a disorder that is characterized by pregnancy-induced hypertension. It has been reported that Annexin A1 (ANXA1) is highly expressed in the plasma of women diagnosed with PE. Therefore, the present study aimed to examine the effect of ANXA1 on PE rats. The PE animal model was constructed in rats using $\mathrm{N} \omega$-nitro-L-arginine methyl ester (L-NAME), and the blood pressure and urine protein levels of rats were detected. The pathological features of placental tissue, and the levels of inflammatory factors and ANXA1 were respectively measured by hematoxylin-eosin staining, enzyme-linked immunosorbent assay and immunohistochemical assay. The activity of trophoblasts obtained from PE placental tissue was measured using immunofluorescence staining, while cell apoptosis was assessed using flow cytometry. The levels of associated factors were determined by reverse transcription-quantitative polymerase chain reaction and western blot analysis. The results identified that systolic blood pressure, diastolic blood pressure, mean arterial pressure and urine protein levels were enhanced, and that the contents of ANXA1, tumor necrosis factor alpha (TNF- $\alpha$ ), interleukin (IL)-1 $\beta$, IL-6 and IL-8 were increased in the L-NAME group. Transfection with small interfering RNA (siRNA)-ANXA1 markedly decreased the apoptosis and inflammatory response of trophoblasts. In addition, siRNA-ANXA1 upregulated the levels of B-cell lymphoma-2 (Bcl-2) and pro-caspase-3, and downregulated the levels of Bcl-2-associated $\mathrm{X}$ protein, cleaved-caspase-3, TNF- $\alpha$, IL-1 $\beta$, IL-6 and IL-8. Furthermore, siRNA-ANXA1 repressed the phosphorylation of Janus kinase 2 (JAK2) and signal transducer and activator of transcription 3 (STAT3); however, siRNA-ANXA1 did not alter the levels of JAK 2 and STAT3. Therefore, silencing of ANXA1
\end{abstract}

Correspondence to: Dr Jing Feng, Department of Gynaecology and Obstetrics, Hebei General Hospital, 348 Heping Road, Shijiazhuang, Hebei 050051, P.R. China

E-mail: jingfeng_fjj@163.com

Key words: preeclampsia, Annexin A1, inflammatory response, Janus kinase 2/signal transducer and activator of transcription 3 pathway suppressed the apoptosis and inflammatory response of PE rat trophoblasts, and downregulated JAK2/STAK3 pathway.

\section{Introduction}

Preeclampsia (PE) is a disorder that is characterized by pregnancy-induced hypertension. The incidence of PE among primiparas is $\sim 8 \%$, and approximately 50,000 mortality cases in pregnant women worldwide are reported annually due to PE and its complications $(1,2)$. PE usually occurs after 20 weeks of gestation, and the main clinical manifestations include hypertension, proteinuria, and placental and renal tissue injury. Severe PE can advance to eclampsia, leading to complications, such as HELLP syndrome (involving hemolysis, increased liver enzyme levels and thrombocytopenia), coagulation dysfunction, cardiopulmonary syndrome and cardio-renal syndrome, which directly threaten the safety of pregnant women $(3,4)$. PE not only produces adverse effects on the maternal body, but also leads to fetal hypoxic-ischemic injury, resulting in intrauterine growth retardation and even fetal death (5-7). Although numerous efforts have been made in order to fully understand PE, the pathogenesis of PE remains unclear. This contributes to the current lack of an effective screening method and treatment of PE; therefore, PE currently remains one of the most refractory diseases in clinical practice. Therefore, it is necessary to explore the pathogenesis of $\mathrm{PE}$, identify novel biological markers for early diagnosis and develop new strategies for treating this disorder.

Annexin A1 (ANXA1), also known as Annexin I, is a member of the calcium-dependent phospholipid-binding protein superfamily of Annexins, which can bind to negatively charged phospholipids (8). Annexin A is usually used to detect cell apoptosis as it can bind to phosphatidylserine, a marker of apoptosis on the outer leaflet of the membrane (9). Annexin A1 is considered to be able to mitigate inflammation by interacting with Annexin A1 receptors (10). ANXA1 is located in chromosome $9 q 24$, which contains 13 exons and 12 introns, and is an anti-inflammatory protein $(11,12)$. It can participate in various cell activities, including the anti-inflammatory response, differentiation and proliferation, cell signal regulation, as well as phagocytosis of apoptotic cells $(13,14)$. Nevertheless, the specific mechanism of ANXA1 in PE is not fully clear.

The Janus kinase 2 (JAK2)/signal transducer and activator of transcription 3 (STAT3) signaling pathway is thought to act as a signal transduction pathway that is activated by cytokines. 
JAK2/STAT3 pathway is associated with the occurrence and development of numerous diseases, such as tumors, PE and bone diseases among others, and it mainly participates in inflammatory response, growth, differentiation and apoptosis of cells (15-18). Extracellular signals or cytokines are able to change the receptor structure by binding to the receptor on the cell membrane, enabling JAK2 to move onto the membrane receptor and activate the tyrosine phosphorylation of JAK2 (19). Next, activated JAK2 phosphorylates STAT3 and these activated STATs formed dimers, then the dimers enter into cell nucleus and regulate the downstream gene expression (20).

In the present study, a model of PE was constructed in rats, and the expression of ANXA1 in the model was detected. Placental trophoblasts were obtained from PE rats, and the roles and molecular mechanism of ANXA1 in these cells were explored. The data revealed that the knockdown of ANXA1 decreased the apoptosis and inflammatory response of PE trophoblasts.

\section{Materials and methods}

Animal model construction and grouping. A total of 20 male and 20 female Sprague-Dawley rats (weight, 250-300 g; age, 7-9 weeks; Guangdong Medical Laboratory Animal Center, Guangzhou, China) were kept in cages at a temperature set between 23 and $26^{\circ} \mathrm{C}$ (50-70\% humidity and 12-h light/dark cycle). The rats had free access to food and water, and the ratio of male to female was 1:1. After mating of the rats, in the following morning, small wet cotton swabs with saline were used to collect vaginal secretions of female rats, and this was recorded as the day 0 of pregnancy (vaginal plugs were used to determine if the rats were pregnant). A hypertensive model group was established by gavaging 6 pregnant rats using $80 \mathrm{mg} / \mathrm{kg} /$ day $\mathrm{N} \omega$-nitro-L-arginine methyl ester (L-NAME; Sigma-Aldrich; Merck KGaA, Darmstadt Germany) for 8 days starting on day 12 of the pregnancy, and this was termed the L-NAME group. In the control group, 6 pregnant rats were gavaged using $80 \mathrm{mg} / \mathrm{kg} / \mathrm{day}$ normal saline for 8 days. All animal tests conducted in the present study were approved by the Ethics Committee of Hebei General Hospital (Shijiazhuang, China).

Detection of blood pressure and urine protein. The systolic blood pressure (SBP), diastolic blood pressure (DBB) and mean arterial pressure (MAP) of rats were detected on days 12, 16 and 20 of pregnancy, using a non-invasive rat tail arterial blood pressure monitor (Tail Cuff Blood Pressure Systems; IITC Life Science, Woodland Hills, CA, USA). The method was as follows: The pregnant rats were placed in a fixator, which was adjusted in terms of the size of the pregnant rat. An air bag was sleeved in the middle of the tail, and the measuring instrument was well-connected. The blood pressure of the rat tail artery was detected when the rat was calm and the pulse wave of the blood pressure measuring instrument was stable. The mean value of each rat was obtained based on five measured values.

In addition, the rats were kept in standard metabolic cages on days 12,16 and 20 of their pregnancy, and their urine volume was collected for $24 \mathrm{~h}$. The 24-h urinary protein (UP) level was tested with an automatic biochemical analyzer (AU5800; Beckman Coulter, Inc., Brea, CA, USA).
Specimen collection. Prior to placental tissue collection, urine was obtained from the rats on day 20 of pregnancy. Under anesthesia by an intraperitoneal injection of chloral hydrate $(350 \mathrm{mg} / \mathrm{kg}$ ), the animals were sacrificed by rapid cervical dislocation (21). The uterus was exposed and cut open, and the placenta was removed. The placenta was fixed using $4 \%$ polymethylene for $24 \mathrm{~h}$. Next, the placenta was embedded in paraffin and prepared for hematoxylin and eosin (H\&E) and immunohistochemical staining.

Following the collection of placental tissue, the abdominal organs and the abdominal viscera were pushed to one side in order to expose the inferior vena cava and the abdominal aorta. An index finger was used to touch the blood vessel, and the abdominal aorta was more evident. The blood of the rats was collected from the bifurcation of the abdominal aorta and centrifuged at $1,500 \mathrm{xg}$ for $10 \mathrm{~min}$ at $4^{\circ} \mathrm{C}$. The serum was then used for enzyme-linked immunosorbent assay (ELISA).

$H \& E$ staining. Paraffin-embedded placental tissue was sliced, and the thickness of each slice was $\sim 4 \mu \mathrm{m}$. The slices were routinely dewaxed with dimethylbenzene for $15 \mathrm{~min}$ and $95 \%$ ethanol for $3 \mathrm{~min}$ at room temperature, and then washed three times with distilled water. Next, the slices were stained by hematoxylin (Beijing Solarbio Science \& Technology Co., Ltd., Beijing, China) for $15 \mathrm{~min}$ at room temperature. The slices were then washed three times with distilled water, followed by treatment with $1 \%$ hydrochloric acid alcohol. The slices were then washed three times with distilled water and stained with eosin staining solution (Beijing Solarbio Science $\&$ Technology Co., Ltd.). Subsequently, the slices were dehydrated using gradient alcohol (80\% ethanol for $2 \mathrm{sec}, 95 \%$ ethanol for $3 \mathrm{~min}$ and absolute alcohol for $6 \mathrm{~min}$ ), and neutral gum (Beijing Solarbio Science \& Technology Co., Ltd.) was used for mounting. Finally, the slices were observed under an inverted fluorescence microscope (MF53; Micro-shot Technology Co., Ltd., Guangzhou, China).

Immunohistochemical assay. The tissue slices were routinely dewaxed, washed with distilled water, soaked in $0.01 \mathrm{M}$ citrate buffer $(\mathrm{pH}=6.0)$ and heated to $95^{\circ} \mathrm{C}$ using a microwave. Subsequent to cooling, the slices were washed using PBS $(\mathrm{pH}=7.4)$, following which $3 \%$ hydrogen peroxide was added to the slices and maintained for $20 \mathrm{~min}$. The slices were then incubated with anti-ANXA1 antibody (cat. no. ab214486; 1:1,000; Abcam, Cambridge, MA, USA), at $4^{\circ} \mathrm{C}$ for $24 \mathrm{~h}$. On the following day, goat anti-mouse IgG secondary antibodies (cat. no. ab6708; 1:8,000; Abcam) were added to the slices at $37^{\circ} \mathrm{C}$ and maintained for $30 \mathrm{~min}$. The slices were then stained with 3,3'-diaminobenzidine solution (Leica Microsystems, Shanghai, China) for $10 \mathrm{~min}$. After washing three times with distilled water, the slices were dyed using hematoxylin for $30 \mathrm{sec}$ at room temperature. The slices were finally observed under an inverted fluorescence microscope.

ELISA. The levels of the inflammatory factors-tumor necrosis factor- $\alpha$ (TNF- $\alpha$ ), interleukin (IL)- $1 \beta$, IL- 6 and IL- 8 were measured using TNF- $\alpha$ ELISA kit (cat. no. RTA00; R\&D Systems, Inc., Minneapolis, MN, USA), IL-1 $\beta$ ELISA kit (cat. no. RLB00; R\&D Systems, Inc.), IL-6 ELISA kit (cat. no. ESK6029; Sangon Biotech Co., Ltd., Shanghai, China) 
Table I. Sequences of primers used in polymerase chain reaction.

\begin{tabular}{llc}
\hline Primer name & \multicolumn{1}{c}{ Sequence $\left(5^{\prime}-3^{\prime}\right)$} & Product size $(\mathrm{bp})$ \\
\hline ANXA1 forward & CTGGAGGAGGTTGTTTTGGC & 238 \\
ANXA1 reverse & GAGCAAGCAAGGCATTACGA & 187 \\
Bax forward & GAGACACCTGAGCTGACCTT & 221 \\
Bax reverse & CGTCTGCAAACATGTCAGCT & 225 \\
Bcl-2 forward & GCCTTCTTTGAGTTCGGTGG \\
Bcl-2 reverse & CTGAGCAGCGTCTTCAGAG & \\
GAPDH forward & AGTCTACTGGCGTCTTCACC & \\
GAPDH reverse & CCACGATGCCAAAGTTGTCA &
\end{tabular}

ANXA1, Annexin A1; Bcl-2, B-cell lymphoma 2; Bax, Bcl-2-associated X protein.

and respectively. All the regents used for the ELISA assay were provided by the kits and the determination was performed according to the manufacture's protocols. The optical density value at $450 \mathrm{~nm}$ was measured using SMR16.1 multimode reader (USCN Business Co., Ltd., Wuhan, China).

Cell culture and transfection. The placental trophoblasts were obtained from the placenta of pregnant rats with hypertension. In brief, the villi tissues were cut into $-1 \mathrm{~mm}^{3}$ pieces and digested with $0.25 \%$ trypsin (Beijing Solarbio Science \& Technology Co., Ltd.) and $0.1 \%$ collagenase (Beijing Solarbio Science \& Technology Co., Ltd.) at $37^{\circ} \mathrm{C}$ for $25 \mathrm{~h}$. The reaction was stopped by addition of the serum. The solution was centrifuged at $1,200 \times \mathrm{g}$ for $30 \mathrm{~min}$ at $4^{\circ} \mathrm{C}$. The collected trophoblasts were cultured in Dulbecco's modified Eagle's medium (DMEM; Invitrogen; Thermo Fisher Scientific, Inc., Waltham, MA, USA) with $10 \%$ fetal bovine serum (FBS; Invitrogen; Thermo Fisher Scientific, Inc.), $100 \mathrm{U} / \mathrm{ml}$ penicillin and $100 \mu \mathrm{g} / \mathrm{ml}$ streptomycin at $37^{\circ} \mathrm{C}$ in an incubator that contained $5 \% \mathrm{CO}_{2}$. The cells were identified under an inverted microscope.

For ANXA1 knockdown, ANXA1 small interfering RNA (siRNA) and empty vectors (30 $\mathrm{nM})$ were purchased from the Genomeditech (Shanghai, China). The vectors were transfected into trophoblasts by Lipofectamine 2000 (Invitrogen; Thermo Fisher Scientific, Inc.).

Immunofluorescence staining. The trophoblasts were isolated from placental tissues and then the expression of vimentin was examined by immunofluorescence staining to identify trophoblasts. Briefly, the villi tissues were homogenized and soaked in Hank's balanced salt solution supplemented with HEPES (25 mmol), DNasel and collagenase (15 U/ml; Sigma-Aldrich; Merck KGaA, Darmstadt, Germany) at $37^{\circ} \mathrm{C}$ with agitation for $30 \mathrm{~min}$. The dispersed cells were separated by filtering through a screen mesh (40-mm diameter per pore). The cells in Ficoll suspension were centrifuged at 1,200 x g for $30 \mathrm{~min}$ at $4^{\circ} \mathrm{C}$, and the obtained cells were maintained in DMEM with $10 \%$ FBS. Subsequent to culturing, the trophoblasts were washed using PBS and fixed with $4 \%$ paraformaldehyde at $4{ }^{\circ} \mathrm{C}$ for $20 \mathrm{~min}$. Goat serum (Beyotime Institute of Biotechnology, Shanghai, China) was then added to block the cells (at room temperature for $30 \mathrm{~min}$ ), followed by incubation with the anti-vimentin primary antibody (cat. no. ab8978; 1:1,000; Abcam) $4^{\circ} \mathrm{C}$ for $24 \mathrm{~h}$. PBS was used as the negative control for the anti-vimentin primary antibody. Next, the cells were incubated using a fluorescence-conjugated secondary antibody (Alexa-Fluor ${ }^{\circledR} 594$ goat anti-rabbit antibody IgG; Thermo Fisher Scientific, Inc., Waltham, MA, USA) for $1.5 \mathrm{~h}$ at room temperature. Nuclei were counterstained by Hoechst 33258 (Beyotime, Shanghai, China), and images were obtained using an inverted fluorescence microscope.

Cell apoptosis. Cell apoptosis was analyzed using an Annexin V-FITC/propidium iodide (PI) apoptosis detection kit (Beijing Solarbio Science \& Technology Co., Ltd.). The trophoblasts were first seeded in a 6 -well plate $\left(5 \times 10^{4}\right.$ cells/well $)$ for $24 \mathrm{~h}$, and then treated with PBS, siRNA-ANXA1 or empty vector using Lipofectamine 2000 (Invitrogen; Thermo Fisher Scientific, Inc.) and incubated at $37^{\circ} \mathrm{C}$ for further $24 \mathrm{~h}$. The cells were digested by trypsin (Beijing Solarbio Science \& Technology Co., Ltd.) and re-suspended with a $1 \mathrm{X}$ binding buffer. Next, the cells were double-stained with Annexin V-FITC/PI for $20 \mathrm{~min}$, and the cell apoptosis was determined using flow cytometry (BD Biosciences, Franklin Lakes, NJ, USA).

Reverse transcription-quantitative polymerase chain reaction (RT-qPCR) assay. The total RNA of cells was isolated using a TRIzol reagent (Promega Corporation, Beijing, China). The concentration of RNA was measured by ultra-micro protein nucleic acid analyzer (NanoDrop; Thermo Fisher Scientific, Inc., Wilmington, DE, USA). In total, $1 \mu \mathrm{g}$ RNA was used for cDNA synthesis by an ABScript II cDNA First Strand synthesis kit (ABclonal Biotech Co., Ltd., Wuhan, China) following the manufacturer's protocols. A SYBR Premix Taq ${ }^{\mathrm{TM}}$ II kit (Dalian Meilun Biology Technology Co., Ltd, Dalian, China) was then used to amplify cDNA, according to the instructions of the kit. The primers used were purchased from BersinBio Co., Ltd. (Guangzhou, China), and are listed in Table I. The data were analyzed with the $2^{-\Delta \Delta C q}$ calculation (22), and the relative mRNA expression levels were normalized to that of GAPDH.

Western blot analysis. The cells were lysed by radioimmunoprecipitation assay buffer (Thermo Fisher Scientific, 
A
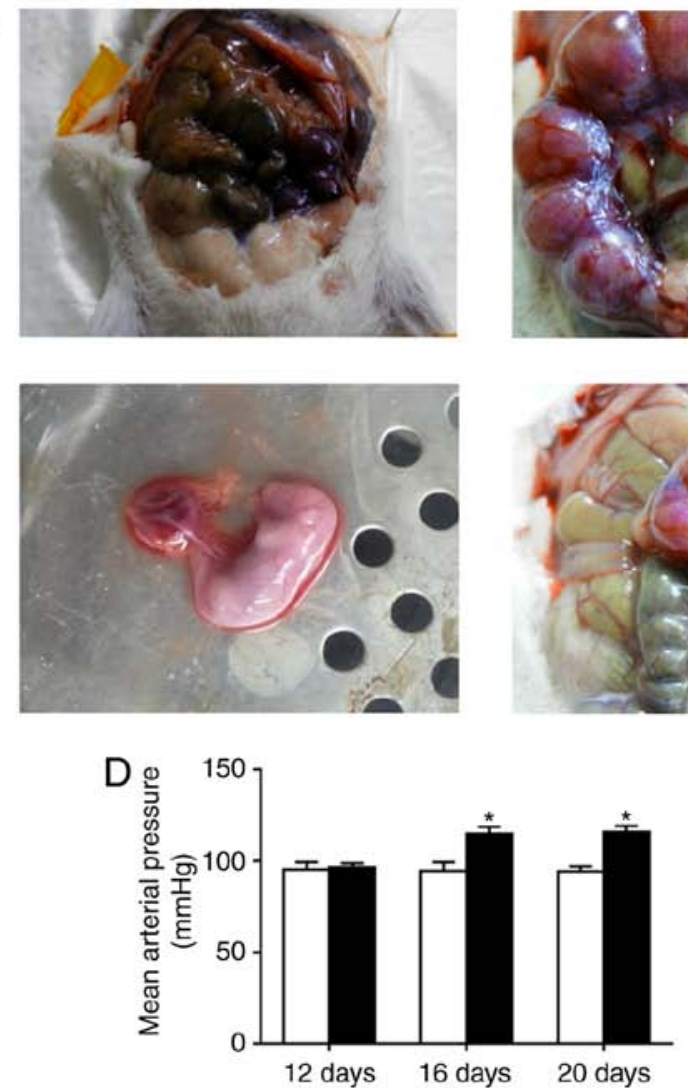
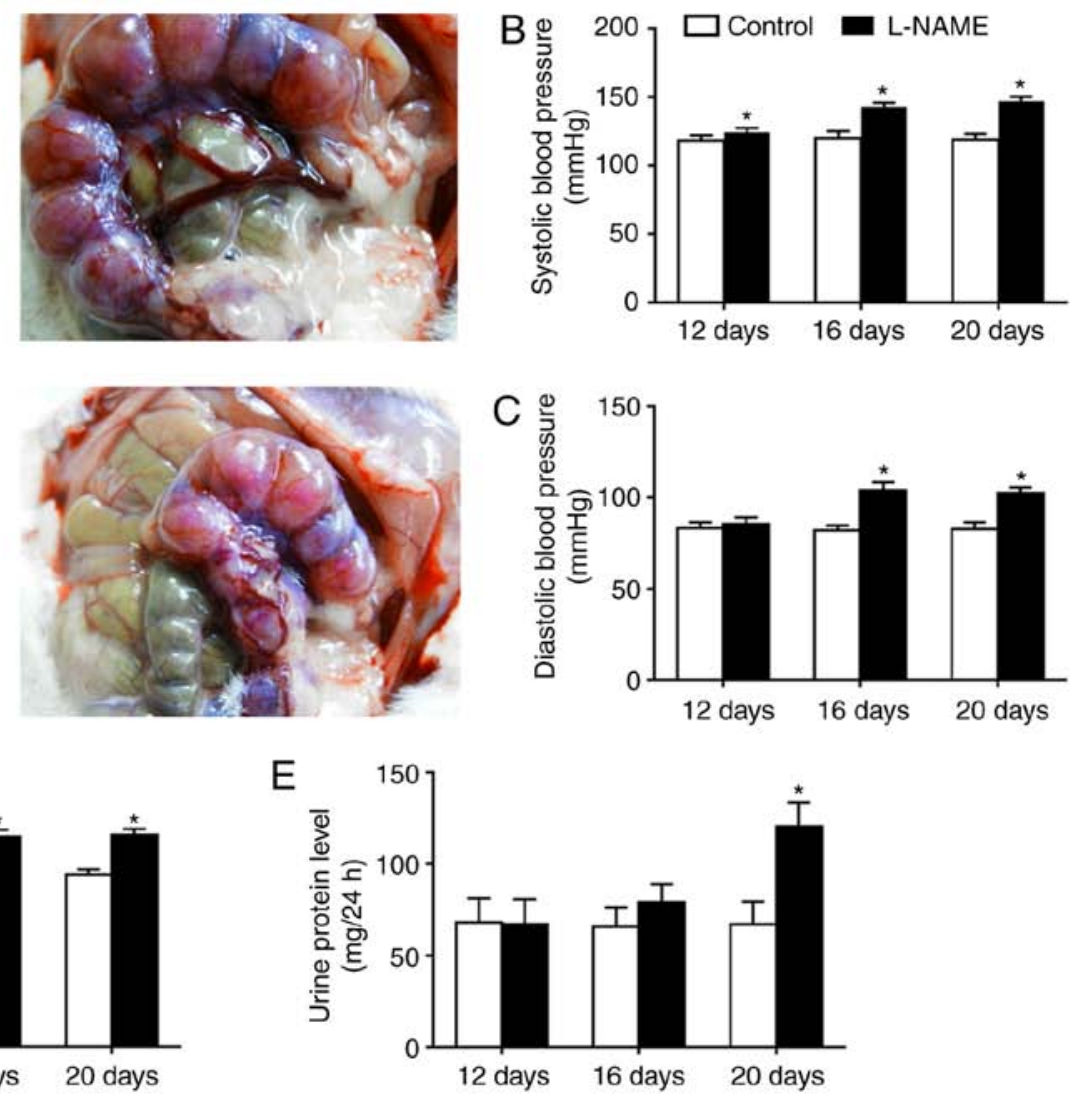

Figure 1. Blood pressure and urinary protein levels were increased in the L-NAME group. (A) The placental tissues obtained from pregnant rats. (B) Systolic blood pressure, (C) diastolic blood pressure and (D) mean arterial pressure were detected on days 12, 16 and 20 of pregnancy using a non-invasive rat tail arterial blood pressure monitor. (E) Urinary protein level was tested by an automatic biochemical analyzer. "P<0.05 vs. control group. L-NAME, N $\omega$-nitro-L-arginine methyl ester.

Inc.). The protein concentrations of samples were measured using a BCA protein assay kit (Qiyi Biological Technology Co., Ltd., Shanghai, China). Next, the proteins $(25 \mu \mathrm{g})$ were separated by $12 \%$ sodium dodecyl sulfate-polyacrylamide gel electrophoresis and then transferred onto a polyvinylidene fluoride membrane (EMD Millipore, Billerica, MA, USA). Tris-buffered saline/Tween 20 buffer containing 5\% skimmed milk was added to block the membranes at room temperature for $2 \mathrm{~h}$. The corresponding primary antibodies were subsequently used to incubate the membranes overnight at $4^{\circ} \mathrm{C}$, including anti-ANXA1 (cat. no. ab214486; 1:1,000; Abcam), anti-pro-caspase-3 (cat. no. ab32499; 1:1,000; Abcam), anti-cleaved-caspase-3 (cat. no. ab2302; 1:700; Abcam), anti-B-cell lymphoma-2 (Bcl-2; cat. no. ab692; 1:800; Abcam), anti-Bcl-2-associated X protein (Bax; cat. no. 2774; 1:1,000; Cell Signaling Technology, Inc., Danvers, MA, USA), anti-JAK2 (cat. no. ab108596; 1:600; Abcam), anti-phosphorylated (p)-JAK2 (cat. no. ab195055; 1:800; Abcam; anti-STAT3 (cat. no. ab119352; 1:1,000; Abcam), anti-p-STAT3 (cat. no. ab32143; 1:800; Abcam) and anti-GAPDH (cat. no. ab8245; 1:600; Abcam). On the following day, the membranes were incubated with the following secondary antibodies at room temperature for $1 \mathrm{~h}$ : Rabbit anti-mouse IgG (cat. no. ab6709; 1:6,000; Abcam), mouse anti-rabbit IgG (cat. no. 93702; 1:8,000, Cell Signaling Technology, Inc.), and goat anti-rabbit (cat. no. ab205718; 1:6,000; Abcam). The blots were subsequently developed using a chemiluminescence substrate kit (BeyoECL Star; Beyotime Institute of Biotechnology, Haimen, China). The results were analyzed by the ECL system (Amersham; GE Healthcare, Chicago, IL, USA).

Statistical analysis. Statistical analysis was conducted using SPSS version 20.0 software (IBM Corporation, Armonk, NY, USA). Data are expressed as the mean \pm standard deviation. Student's t-test was used to compare the differences between two groups, while one-way analysis of variance and the post-hoc Dunnett's test were performed to compare the differences among more than two groups. $\mathrm{P}<0.05$ was considered to denote a statistically significant difference.

\section{Results}

Blood pressure and UP levels are increased in the L-NAME group. The uterus and placenta were examined, and serum was obtained (Fig. 1A). The results revealed that the SBP, DBP, MAP and UP levels remained stable on day 12 of the rat pregnancy in the L-NAME group, as compared with those in the control group. However, the SBP, DBP, MAP and UP levels in the L-NAME group were significantly increased in comparison with those in the control group on day 20 of the pregnancy $(\mathrm{P}<0.05$; Fig. 1B-E). These results demonstrated that the PE model was successfully established in the L-NAME-treated rats. 
A
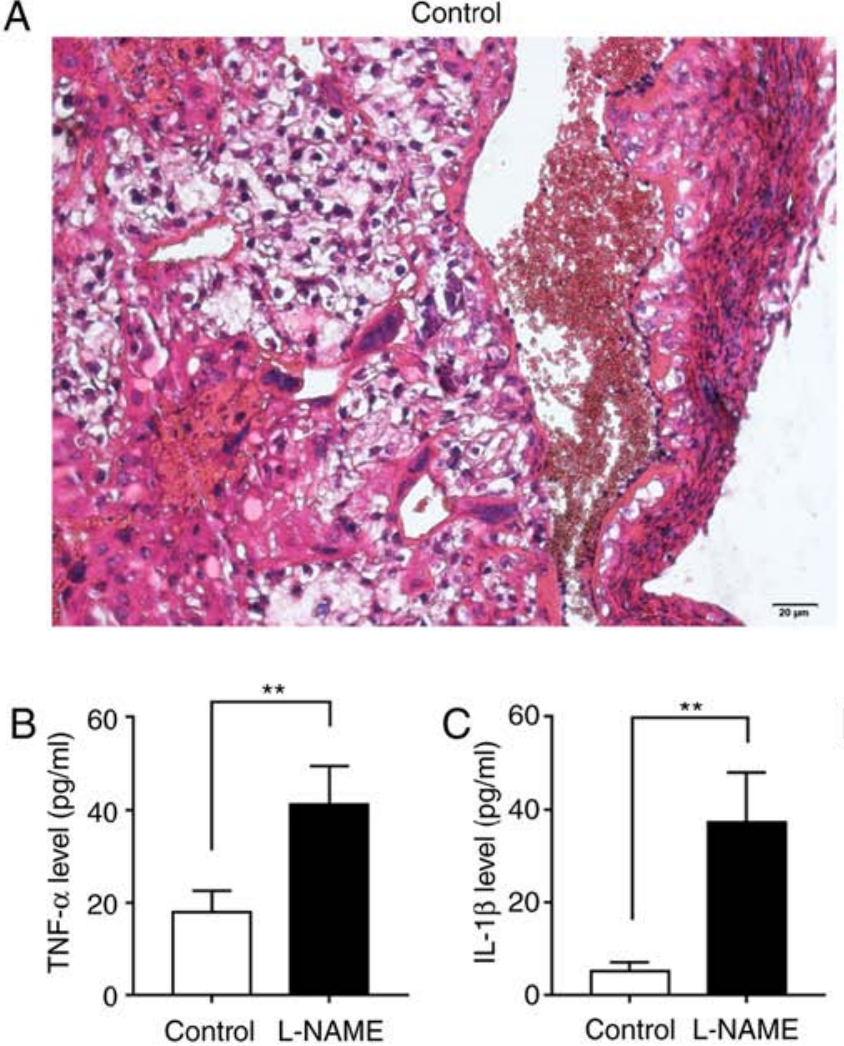

$\mathrm{F}$

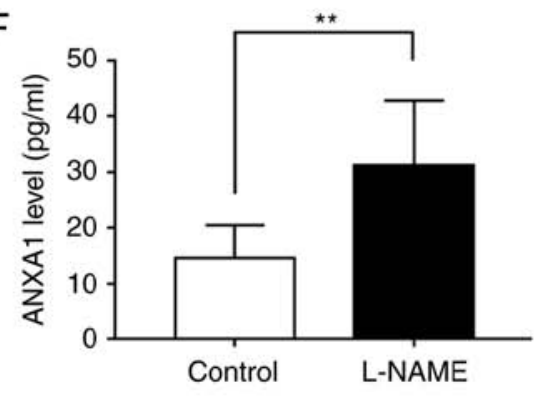

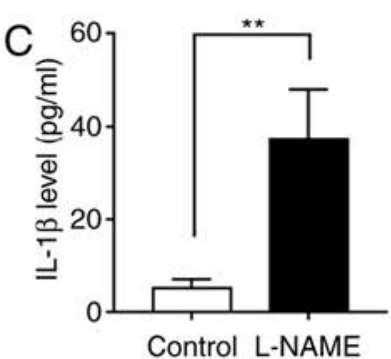

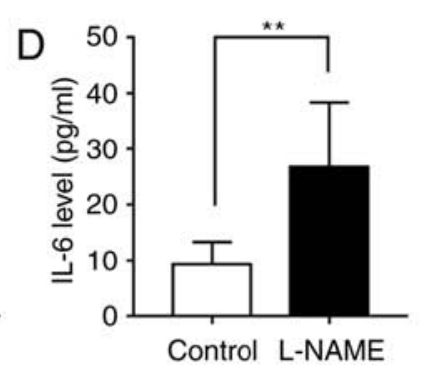

L-NAME
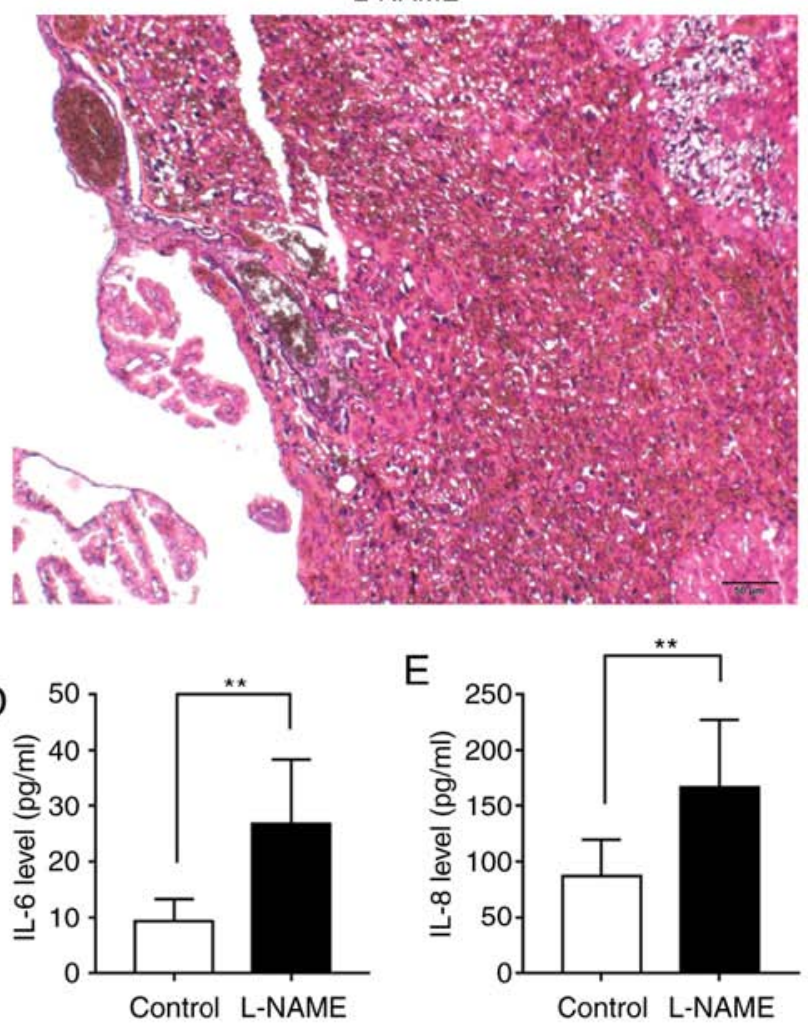

G

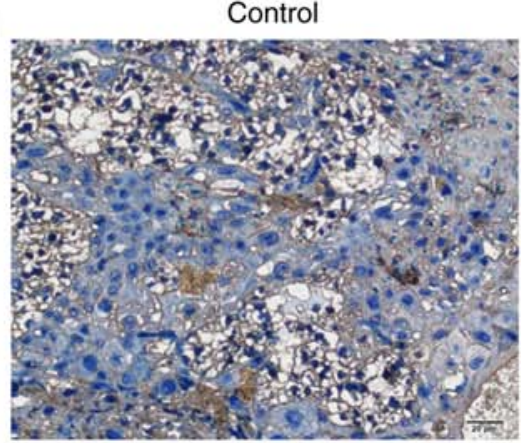

L-NAME

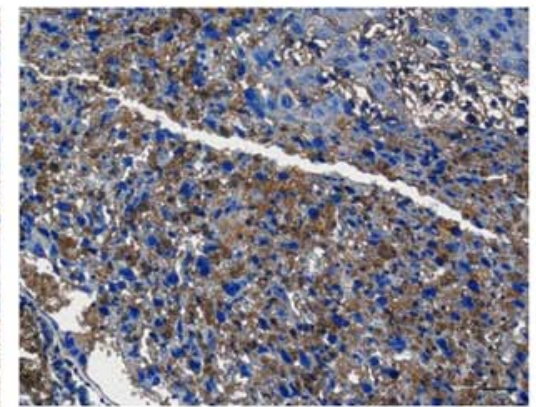

Figure 2. Inflammatory response was observed and ANXA1 expression was upregulated in the L-NAME group. (A) Hematoxylin and eosin staining in the normal (control) and model (L-NAME) tissues. (B) TNF- $\alpha$, (C) IL-1 $\beta$, (D) IL-6 and (E) IL-8 and in the serum were detected using enzyme-linked immunosorbent assay. (F and G) ANXA1 expression was analyzed by an immunohistochemical assay. ${ }^{* *} \mathrm{P}<0.01$, vs. control group. ANXA1, Annexin A1; L-NAME, $\mathrm{N} \omega$-nitro-L-arginine methyl ester; TNF, tumor necrosis factor; IL, interleukin.

Inflammatory response and upregulated ANXA1 expression are observed in the L-NAME group. H\&E staining, ELISA and immunohistochemical assay were performed to investigate the histopathological changes, inflammatory reaction and ANXA1 expression in placental tissues. The H\&E staining images displayed that, in comparison with the control group, the trophoblast proliferation and inflammatory cell infiltration were evidently increased in the L-NAME group (Fig. 2A). The ELISA data revealed that the levels of TNF- $\alpha$, IL-1 $\beta$, IL- 6 and IL-8 in L-NAME group were higher as compared with those in the control group (Fig. 2B-E; $\mathrm{P}<0.01$ ). The immunohistochemical assay results also revealed that there was a considerable amount of brown particles in the L-NAME group, which represented positive expression of ANXA1 (Fig. 2F and G). These results indicated that the expression of inflammatory factors and ANXA1 was upregulated during PE.
Knockdown of ANXA1 inhibits apoptosis and inflammatory response in trophoblasts. The trophoblasts were identified by staining with vimentin antibody (Fig. 3). The RT-qPCR and western blotting data demonstrated that the mRNA and protein levels of ANXA1 were reduced in cells transfected with siRNA-ANXA1 as compared with those transfected with empty vector (Fig. 4A and $\mathrm{B} ; \mathrm{P}<0.01$ ). The flow cytometry results also observed that the apoptosis rate was significantly decreased when cells were transfected with siRNA-ANXA1 (Fig. 4C; $\mathrm{P}<0.01$ ). As displayed by the ELISA data, siRNA-ANXA1 markedly suppressed the levels of TNF- $\alpha$, IL-1 $\beta$, IL-6 and IL-8 (Fig. 4D-G; P<0.05). Therefore, downregulation of ANXA1 decreased the apoptosis during PE.

Knockdown of ANXA1 regulates the levels of apoptosis-associated factors in trophoblasts. Bcl-2 and Bax are 

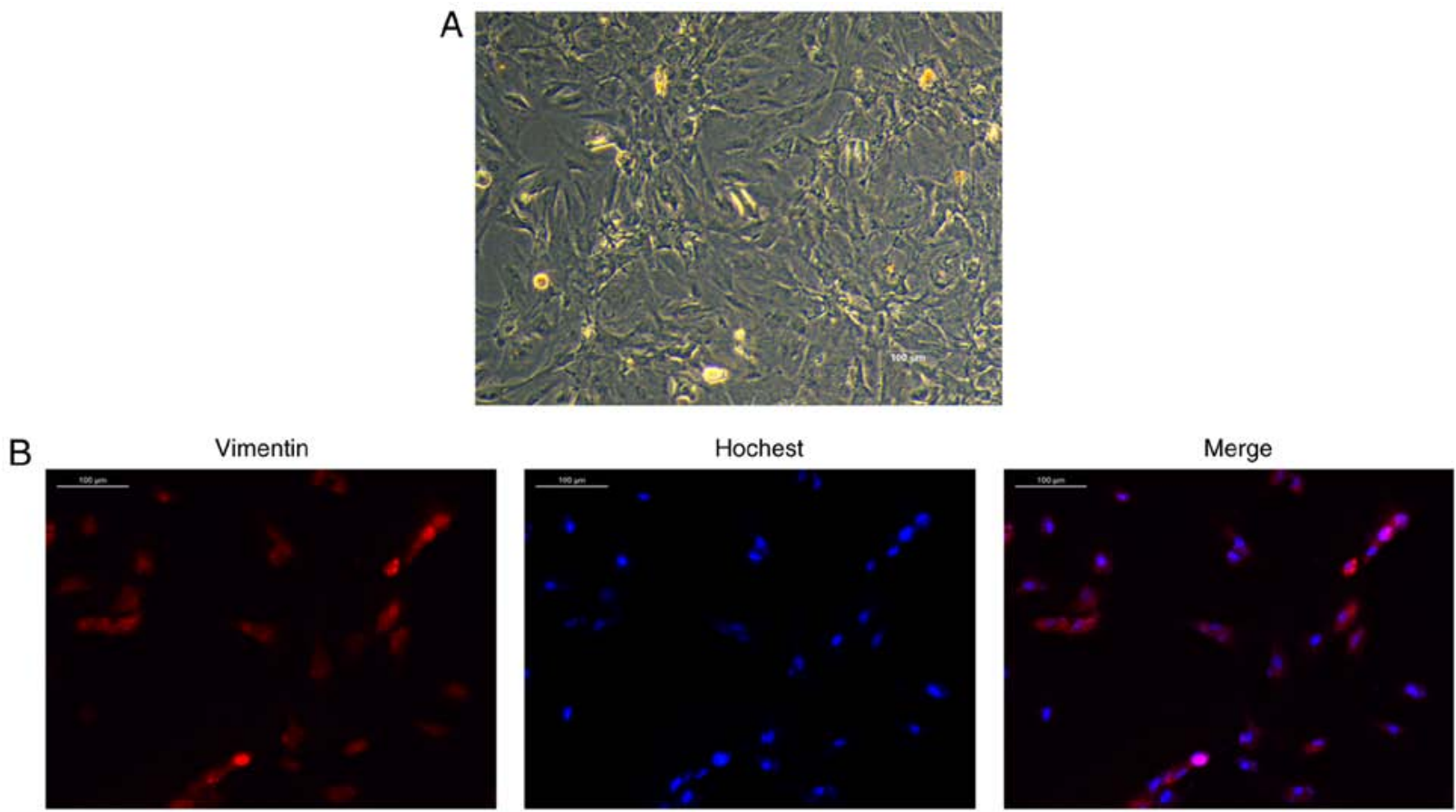

Figure 3. Trophoblasts isolated from the L-NAME group were verified. (A) The morphology of the trophoblasts in the L-NAME group was observed under an inverted fluorescence microscope. (B) Immunofluorescence staining was conducted to identify the activities of vimentin and nucleus in trophoblasts. L-NAME, N $\omega$-nitro-L-arginine methyl ester.

known to regulate the cell apoptosis (23), while caspase-3 is activated by multiple apoptotic signals by cleaving pro-caspase- 3 into cleaved-caspase-3 (24). Therefore, RT-qPCR and western blot analysis were performed to explore the effect of ANXA1 on these apoptosis-associated factors in trophoblasts. As demonstrated by the RT-qPCR data, siRNA-ANXA1 transfection significantly decreased Bax mRNA expression and increased Bcl-2 mRNA expression (Fig. 5A and B; P<0.05). Furthermore, the western blot analysis data revealed that siRNA-ANXA1 promoted the expression levels of pro-caspase- 3 and $\mathrm{Bcl}-2$ protein, whereas it reduced the expression levels of cleaved-caspase-3 and Bax protein (Fig. 5C; $\mathrm{P}<0.01$ ). Therefore, downregulation of ANXA1 inhibited the apoptosis during PE through regulating the expression of apoptosis-related genes.

Knockdown of ANXA1 suppresses the JAK2/STAT3 signaling pathway in trophoblasts. JAK2/STAT3 pathway was examined using western blot analysis to help investigate the pathway of ANXA1 in trophoblasts. The data revealed that the phosphorylation of JAK2 and STAT3 was downregulated in siRNA-ANXA1-treated cells as compared with the empty vector group. The levels of JAK2 and STAT3 remained stable in all groups (Fig. 6; $\mathrm{P}<0.01$ ). Therefore, the inhibition of JAK2/STAT3 signaling pathway may be related to the effect produced by si-ANXA1.

\section{Discussion}

L-NAME is an inhibitor of nitric oxide (NO) synthase $(25,26)$. $\mathrm{NO}$ is produced by vascular endothelial cells, and its functions include the dilation of blood vessels, and regulation of blood vessel tension and of the cardiovascular system during pregnancy $(27,28)$. Increasing evidence suggested that L-NAME can be used as an inducer of PE symptoms in pregnant rats (29-31). In the present study, it was observed that SBP, DBP, MAP and UP level in the L-NAME group were higher compared with those in the control group. Therefore, this suggested that the PE model was successfully established in pregnant rats using L-NAME.

The placenta is a unique temporary organ during pregnancy that serves an indispensable role in maintaining a normal and stable pregnancy, and preventing pregnancy-associated diseases (32-34). Placental tissue has been widely used as a research object for studying PE $(35,36)$. Therefore, in the current study, normal and PE placental tissues were obtained from rats. Furthermore, previous studies have reported that excessive inflammatory response is an important factor leading to the onset of PE (37-39). Several inflammatory factors, including TNF- $\alpha$, IL-1 $\beta$, IL-6 and IL-8, are suggested to contribute to the development of PE. Hence, the levels of these factors in the placental tissues were detected in the present study. The results demonstrated that TNF- $\alpha$, IL-1 $\beta$, IL- 6 and IL- 8 levels in the L-NAME group were higher compared with those in the control group. These observations indicated that there was a strong inflammatory response in PE placental tissue.

ANXA1 was demonstrated to exhibit various anti-inflammatory properties (40). Researchers have reported that $\mathrm{ANXA} 1_{\mathrm{Ac} 2-26}$ peptide reduced the inflammatory response in ARPE-19 cells and peritonitis rats $(41,42)$. However, ANXA1 is also modulated by pro-inflammatory proteins, suggesting that it may act as a 'brake' in controlling the inflammatory response (43). According to previous studies, ANXA1 expression was enhanced in PE patients $(44,45)$. The increased concentration of ANXA1 may be a compensatory mechanism underlying systemic inflammation (45). Similarly, the data of the present study displayed 

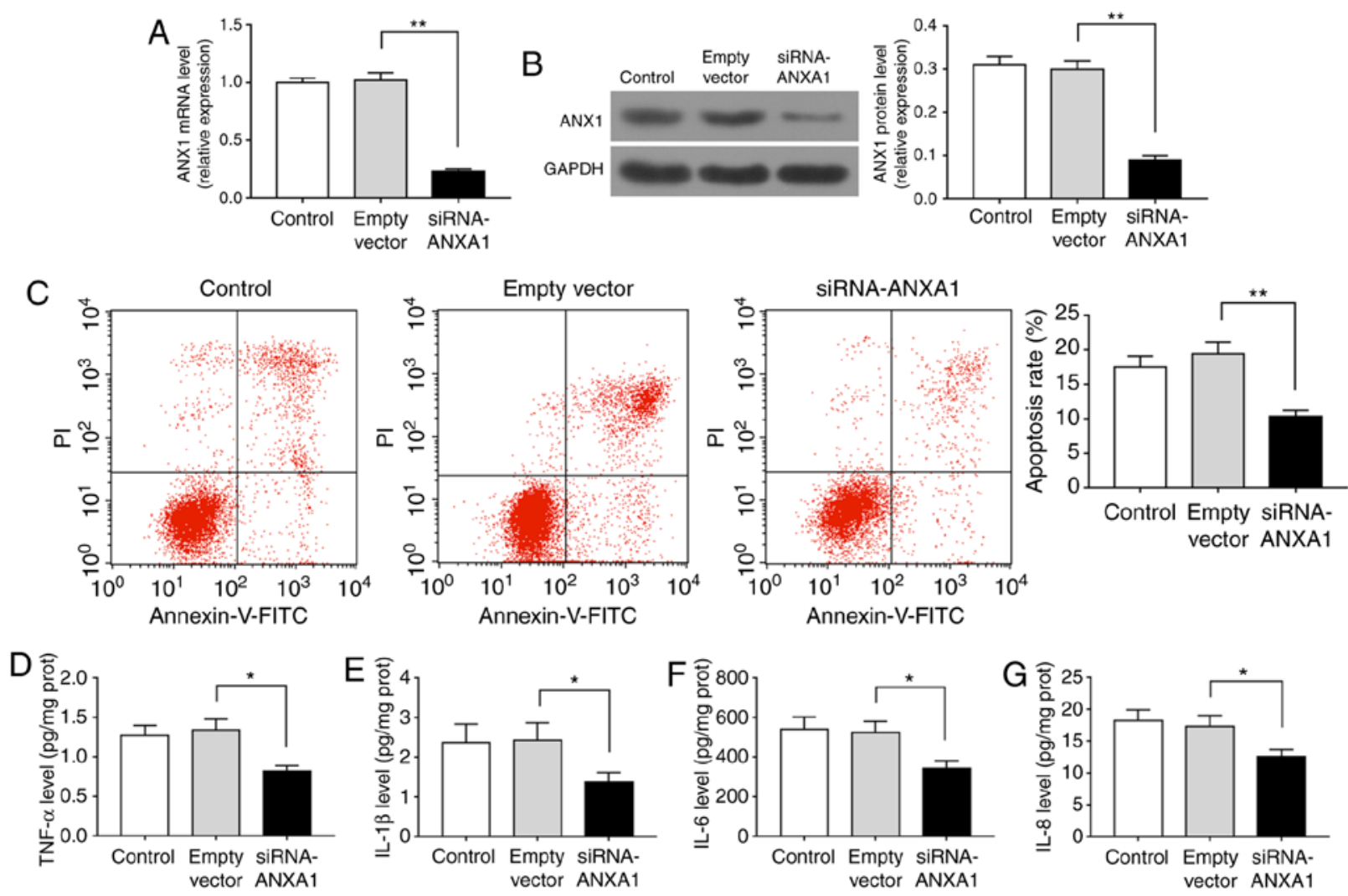

Figure 4. Knockdown of ANXA1 inhibited apoptosis and inflammatory response in trophoblasts. The trophoblasts were treated with PBS (control), siRNA-ANXA1 and empty vector. (A) Reverse transcription-quantitative polymerase chain reaction and (B) western blot analysis were conducted to detect the level of ANXA1. (C) Cell apoptosis was assessed by flow cytometry. (D) TNF- $\alpha$, (E) IL-1 $\beta$, (F) IL-6 and (G) IL-8 levels were determined using enzyme-linked immunosorbent assay. ${ }^{*} \mathrm{P}<0.05$ and ${ }^{* *} \mathrm{P}<0.01$, vs. empty vector group. ANXA1, Annexin A1; siRNA, small interfering RNA; TNF, tumor necrosis factor; IL, interleukin.

that ANXA1 level in the L-NAME group was higher compared with that of the control group. In order to explore the effect of ANXA1 on PE, the trophoblasts from PE placental tissue were transfected with siRNA-ANXA1. It was observed that the ANXA1 expression in the siRNA-ANXA1 group was lower in comparison with that in the group transfected with empty vector. These results indicated that the transfection efficiency of siRNA-ANXA1 in the trophoblasts was high. It was also observed that siRNA-ANXA1 inhibited the inflammatory response of trophoblasts through decreasing the levels of TNF- $\alpha$, IL-1 1 , IL- 6 and IL-8. Consistently, a previous study demonstrated that transfection with ANXA1 short hairpin RNA inhibited IL- $1 \beta$ expression in ischemia/reperfusion-induced retinal ganglion cells (46).

The inflammatory response during PE alters the inflammation microenvironment, and such a change leads to the trophoblasts being more susceptible to apoptosis (47). In addition, $\mathrm{PE}$ is associated with abnormal lipid metabolism (48), and apoptosis of trophoblasts may be induced by oxidative stress during PE (49). PE is usually considered to be associated with insufficient perfusion of the uterus and placenta from the mother, which contributes to the ischemic and hypoxic microenvironment of placental trophoblasts (49). According to a previous study (49), the apoptosis rate of a trophoblast cell line reached nearly $40 \%$ when the cells were cultured in hypoxic conditions. It has also been reported that ANXA1 enhanced the apoptosis of retinal ganglion cells (46).
Furthermore, Huang et al (50) proved that the knockdown of ANXA1 decreased ischemia/reperfusion-induced apoptosis in nasopharyngeal carcinoma cells. In the current study, ANXA1 silencing noticeably suppressed the apoptosis of the trophoblasts. Subsequently, the apoptosis-associated factors were examined by performing RT-qPCR and western blot analysis. The data revealed that siRNA-ANXA1 markedly enhanced the expression levels of pro-caspase- 3 and $\mathrm{Bcl}-2$, while it reduced the expression levels of cleaved-caspase-3 and Bax, and these results are consistent with those reported in previous studies $(51,52)$. Thus, the data suggested that deletion of ANXA1 suppressed the apoptosis of the trophoblasts by upregulating pro-caspase- 3 and $\mathrm{Bcl}-2$, and downregulating cleaved-caspase-3 and Bax.

JAK2/STAT3 pathway contributes to development of complications in pregnancy. A previous study reported that 5-hydroxytryptamine 2A receptor activated JAK2/STAT3 pathway in human choriocarcinoma cells (17). IL-1 was observed to be able to regulate the invasion of trophoblasts via controlling the phosphorylation of JAK2 and STAT3 (53). Hence, it can be hypothesized that ANXA1 regulates the JAK2/STAT3 pathway in trophoblasts. As expected, the present study identified that siRNA-ANXA1 significantly downregulated the phosphorylation of JAK2 and STAT3, but did not affect the levels of JAK2 and STAT3. This verified that silencing of ANXA1 inhibited the regulation of JAK2/STAT3 pathway in trophoblasts. 

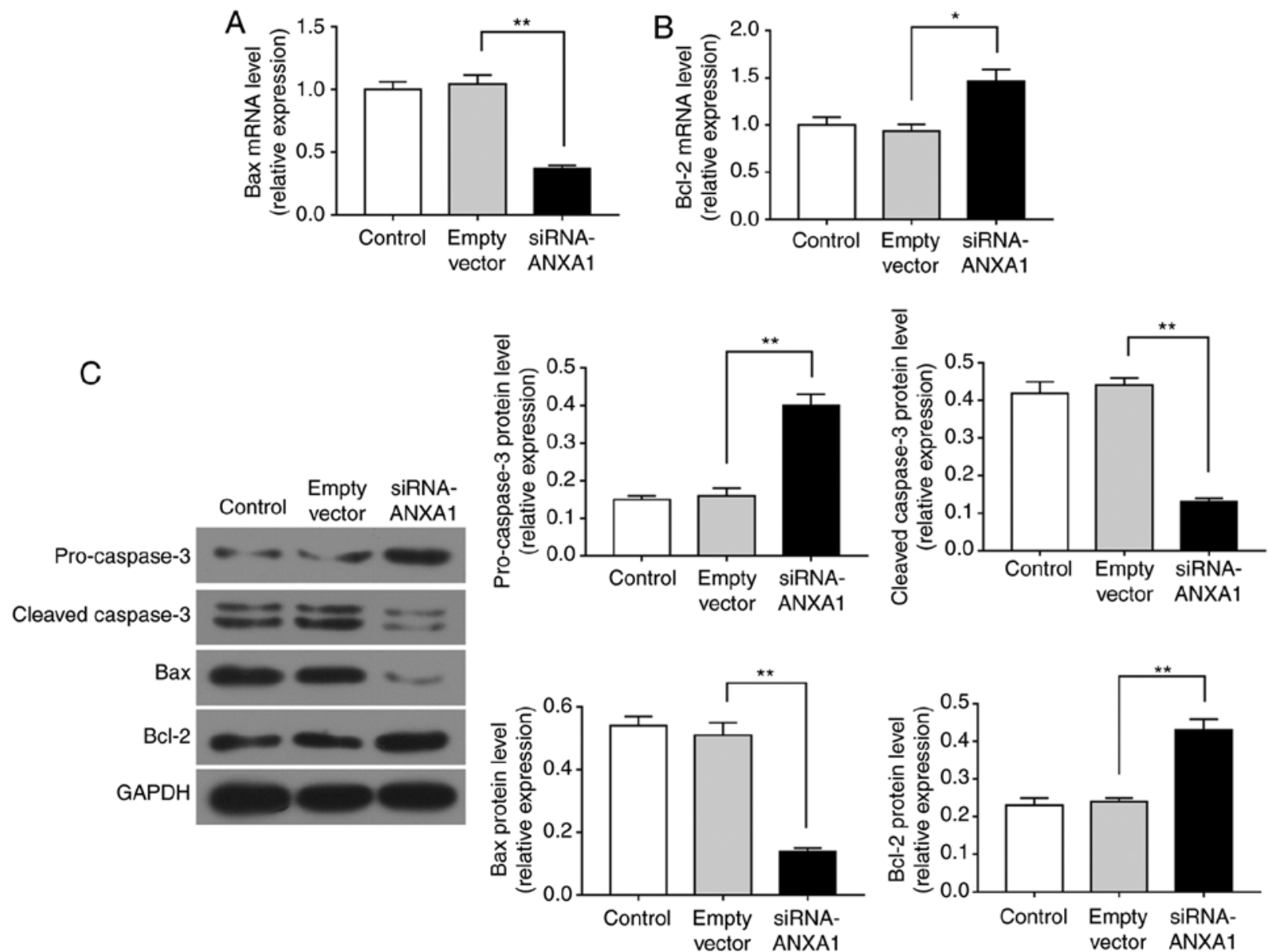

Figure 5. Knockdown of ANXA1 regulated the apoptosis-associated factors in trophoblasts. (A) Bax and (B) Bcl-2 mRNA expression levels were detected using reverse transcription-quantitative polymerase chain reaction. (C) Protein expression levels of pro-caspase-3, cleaved-caspase-3, Bax and Bcl-2 were measured using western blot analysis. ${ }^{* * *} \mathrm{P}<0.01$, vs. empty vector group. ANXA1, Annexin A1; siRNA, small interfering RNA; Bcl-2, B-cell lymphoma 2; Bax, Bcl-2-associated X protein.
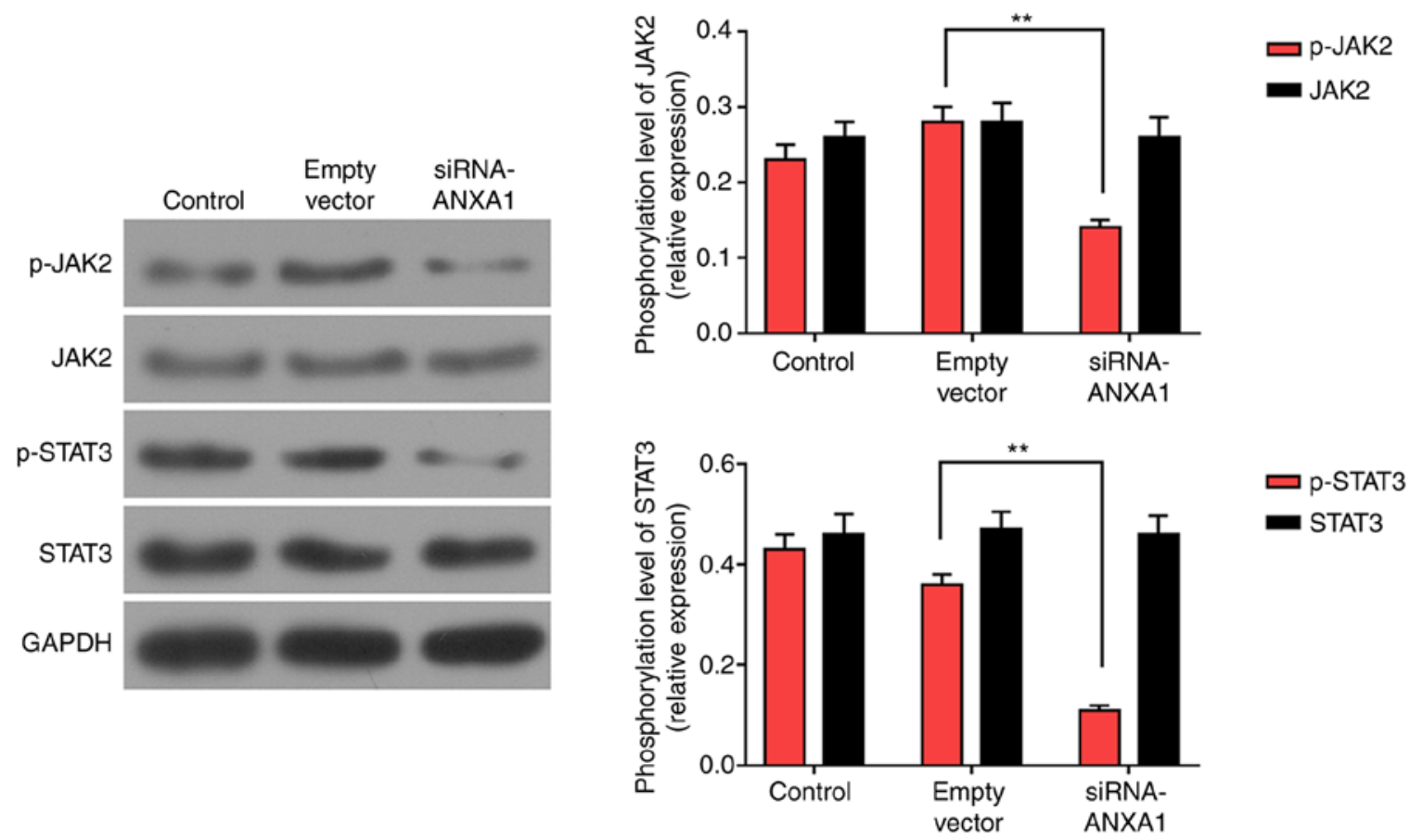

Figure 6. Knockdown of ANXA1 suppressed the JAK2/STAT3 pathway. The protein expression level of p-JAK2, JAK2, p-STAT3 and STAT3 were determined using western blot analysis. ${ }^{*} \mathrm{P}<0.05$ and ${ }^{* *} \mathrm{P}<0.01$, vs. empty vector group. ANXA1, Annexin A1; siRNA, small interfering RNA; JAK2, Janus kinase 2; STAT3, signal transducer and activator of transcription 3; p-, phosphorylated. 
In conclusion, in the current study, an L-NAME-induced model of PE was constructed in rats. The inflammatory response and the increase of ANXA1 expression were observed in the PE model. Silencing of ANXA1 decreased the apoptosis and inflammatory response of trophoblasts obtained from PE placental tissue and downregulated the JAK2/STAK3 pathway. The aforementioned results revealed that ANXA1 may contribute to the pathological mechanism of PE and provided a solid foundation for further study on the specific mechanism of ANXA1 on PE in vivo.

\section{Acknowledgements}

Not applicable.

\section{Funding}

Not applicable.

\section{Availability of data and materials}

The analyzed data sets generated during the study are available from the corresponding author on reasonable request.

\section{Authors' contributions}

JF wrote the main manuscript. JF, XW, HL and LW performed the experiments. QQ, JF and ZT designed the study. XW, HL and $\mathrm{LW}$ performed data analysis. JF, XW, HL, LW and ZT contributed to manuscript revisions. All authors reviewed, read and approved the final manuscript.

\section{Ethics approval and consent to participate}

All animal tests conducted in the present study were approved by the Ethics Committee of Hebei General Hospital (Shijiazhuang, China).

\section{Patient consent for publication}

Not applicable.

\section{Competing interests}

The authors declare that they have no competing interests.

\section{References}

1. Roberts JM and Cooper DW: Pathogenesis and genetics of pre-eclampsia. Lancet 357: 53-56, 2001.

2. Singh SK and Bhatia K: Ultrasonographic optic nerve sheath diameter as a surrogate measure of raised intracranial pressure in severe pregnancy-induced hypertension patients. Anesth Essays Res 12: 42-46, 2018.

3. Contini C, Jansen M, König B, Markfeld-Erol F, Kunze M, Zschiedrich S, Massing U, Merfort I, Prömpeler H, Pecks U, et al: Lipoprotein turnover and possible remnant accumulation in preeclampsia: Insights from the Freiburg Preeclampsia H.E.L.P.-apheresis study. Lipids Health Dis 17: 49, 2018.

4. Kaitu'u-Lino TJ, Brownfoot FC, Beard S, Cannon P, Hastie R, Nguyen TV, Binder NK, Tong $S$ and Hannan NJ: Combining metformin and esomeprazole is additive in reducing sFlt-1 secretion and decreasing endothelial dysfunction-implications for treating preeclampsia. PLoS One 13: e0188845, 2018.
5. Asgharnia M, Mirblouk F, Kazemi S, Pourmarzi D, Mahdipour Keivani M and Dalil Heirati SF: Maternal serum uric acid level and maternal and neonatal complications in preeclamptic women: A cross-sectional study. Int J Reprod Biomed (Yazd) 15: 583-588, 2017.

6. Maruotti GM, Giudicepietro A, Saccone G, Castaldo G, Sarno L, Zullo F, Berghella V and Martinelli P: Risk of preeclampsia in of women who underwent chorionic villus sampling. J Matern Fetal Neonatal Med: 1-4, 2018.

7. Wang F, Fan F, Wang L, Ye W, Zhang Q and Xie S: Maternal cadmium levels during pregnancy and the relationship with preeclampsia and fetal biometric parameters. Biol Trace Elem Res: Apr 12, 2018 (Epub ahead of print).

8. Gerke V and Moss SE: Annexins: From structure to function. Physiol Rev 82: 331-371, 2002.

9. Van ME, Nieland LJ, Ramaekers FC, Schutte B and Reutelingsperger CP: Annexin V-affinity assay: A review on an apoptosis detection system based on phosphatidylserine exposure. Cytometry 31: 1-9, 1998.

10. Hannon R, Croxtall JD, Getting SJ, Roviezzo F, Yona S Paul-Clark MJ, Gavins FN, Perretti M, Morris JF, Buckingham JC and Flower RJ: Aberrant inflammation and resistance to glucocorticoids in annexin 1-/- mouse. FASEB J 17: 253-255, 2003

11. Ansari J, Kaur G and Gavins FNE: Therapeutic potential of annexin A1 in ischemia reperfusion injury. Int J Mol Sci 19: E1211, 2018.

12. Sheikh MH and Solito E: Annexin A1: Uncovering the many talents of an old protein. Int J Mol Sci 19: E1045, 2018.

13. Alli-Shaik A, Wee S, Lim LHK and Gunaratne J: Phosphoproteomics reveals network rewiring to a pro-adhesion state in annexin-1-deficient mammary epithelial cells. Breast Cancer Res 19: 132, 2017.

14. Lee SH, Lee PH, Kim BG, Seo HJ, Baek AR, Park JS, Lee JH, Park SW, Kim DJ, Park CS and Jang AS: Annexin A1 in plasma from patients with bronchial asthma: Its association with lung function. BMC Pulm Med 18: 1, 2018.

15. Bi J, Sun K, Wu H, Chen X, Tang H and Mao J: PPARgamma alleviated hepatocyte steatosis through reducing SOCS3 by inhibiting JAK2/STAT3 pathway. Biochem Biophys Res Commun 498: 1037-1044, 2018.

16. Li CH, Xu LL, Jian LL, Yu RH, Zhao JX, Sun L, Du GH and Liu XY: Stattic inhibits RANKL-mediated osteoclastogenesis by suppressing activation of STAT3 and NF-kappaB pathways. Int Immunopharmacol 58: 136-144, 2018.

17. Oufkir T, Arseneault M, Sanderson JT and Vaillancourt C: The 5-HT 2A serotonin receptor enhances cell viability, affects cell cycle progression and activates MEK-ERK1/2 and JAK2-STAT3 signalling pathways in human choriocarcinoma cell lines. Placenta 31: 439-447, 2010.

18. Sun YX, Zhang HY, Wei YM, Zhu F, Wang M and Liao YH: The mechanism of signal transduction during vascular smooth muscle cell proliferation induced by autoantibodies against angiotensin AT1 receptor from hypertension. Chin Med J (Engl) 121: 43-48, 2008.

19. Parganas E, Wang D, Stravopodis D, Topham DJ, Marine JC, Teglund S, Vanin EF, Bodner S, Colamonici OR, van Deursen JM, et al: Jak2 is essential for signaling through a variety of cytokine receptors. Cell 93: 385-395, 1998.

20. Schindler C, Levy DE and Decker T: JAK-STAT signaling: From interferons to cytokines. J Biol Chem 282: 20059-20063, 2007.

21. Nagy A, Gertsenstein M, Vintersten K and Behringer R: Quick and humane sacrifice of a mouse by cervical dislocation. $\mathrm{CSH}$ Protoc 2006: 2006.

22. Kenneth J and Livak TD: Analysis of relative gene expression data using real-time quantitative PCR and the 2(-Delta Delta C(T)) Method. Method 25: 402-408, 2001.

23. Knudson CM and Korsmeyer SJ: Bcl-2 and Bax function independently to regulate cell death. Nat Genet 16: 358-363, 1997.

24. Salvesen GS: Caspases: Opening the boxes and interpreting the arrows. Cell Death Differ 9: 3-5, 2002.

25. Paredes MD, Romecin P, Atucha NM, O'Valle F, Castillo J, Ortiz MC and García-Estañ J: Beneficial effects of different flavonoids on vascular and renal function in L-NAME hypertensive rats. Nutrients 10: E484, 2018.

26. Vimalraj S, Bhuvaneswari S, Lakshmikirupa S, Jyothsna G and Chatterjee S: Nitric oxide signaling regulates tumor-induced intussusceptive-like angiogenesis. Microvasc Res 119: 47-59, 2018.

27. Osol G, Ko NL and Mandala M: Altered endothelial nitric oxide signaling as a paradigm for maternal vascular maladaptation in preeclampsia. Curr Hypertens Rep 19: 82, 2017. 
28. Palei AC, Spradley FT and Granger JP: Role of nitric oxide synthase on blood pressure regulation and vascular function in pregnant rats on a high-fat diet. Am J Hypertens 30: 240-248, 2017.

29. Acurio J, Herlitz K, Troncoso F, Aguayo C, Bertoglia P and Escudero $\mathrm{C}$ : Adenosine A2A receptor regulates expression of vascular endothelial growth factor in feto-placental endothelium from normal and late-onset pre-eclamptic pregnancies. Purinergic Signal 13: 51-60, 2017.

30. Amaral TAS, Ognibene DT, Carvalho LCRM, Rocha APM, Costa CA, Moura RS and Resende AC: Differential responses of mesenteric arterial bed to vasoactive substances in L-NAME-induced preeclampsia: Role of oxidative stress and endothelial dysfunction. Clin Exp Hypertens 40: 126-135, 2018.

31. Baijnath S, Murugesan S, Mackraj I, Gathiram P and Moodley J: The effects of sildenafil citrate on urinary podocin and nephrin mRNA expression in an L-NAME model of pre-eclampsia. Mol Cell Biochem 427: 59-67, 2017.

32. Deng Q, Liu X, Yang Z and Xie L: Expression of $\mathrm{N}$-Acetylglucosaminyltransferase III promotes trophoblast invasion and migration in early human placenta. Reprod Sci: Jan 1, 2018 (Epub ahead of print).

33. Sahay AS, Jadhav AT, Sundrani DP, Wagh GN, Mehendale SS and Joshi SR: Matrix metalloproteinases-2 (MMP-2) and matrix metalloproteinases-9 (MMP-9) are differentially expressed in different regions of normal and preeclampsia placentae. J Cell Biochem 119: 6657-6664, 2018.

34. Yang A, Xiao XH, Wang ZL, Wang ZY and Wang KY: T2-weighted balanced steady-state free procession MRI evaluated for diagnosing placental adhesion disorder in late pregnancy. Eur Radiol 28: 3770-3778, 2018.

35. Luo S, Pei J, Li X and Gu W: Decreased expression of JHDMID in placenta is associated with preeclampsia through HLA-G. J Hum Hypertens 32: 448-454, 2018.

36. Zardoya-Laguardia P, Blaschitz A, Hirschmug1 B, Lang I, Herzog SA, Nikitina L, Gauster M, Häusler M, Cervar-Zivkovic M Karpf E, et al: Endothelial indoleamine 2,3-dioxygenase-1 regulates the placental vascular tone and is deficient in intrauterine growth restriction and pre-eclampsia. Sci Rep 8: 5488, 2018.

37. Redman CW, Sacks GP and Sargent IL: Preeclampsia: An excessive maternal inflammatory response to pregnancy. Am J Obstet Gynecol 180: 499-506, 1999.

38. Mihu D, Razvan C, Malutan A and Mihaela C: Evaluation of maternal systemic inflammatory response in preeclampsia. Taiwan J Obstet Gynecol 54: 160-166, 2015.

39. Mazouni C, Capo C, Ledu R, Honstettre A, Agostini A, Capelle M, Mege JL and Bretelle F: Preeclampsia: Impaired inflammatory response mediated by toll-like receptors. J Reprod Immunol 78: 80-83, 2008.

40. Parente L and Solito E: Annexin 1: More than an anti-phospholipase protein. Inflamm Res 53: 125-132, 2004

41. Cardin LT, Sonehara NM, Mimura KK, Ramos Dinarte Dos Santos A, da Silva WA Junior, Sobral LM, Leopoldino AM, da Cunha BR, Tajara EH, Oliani SM and Rodrigues-Lisoni FC: ANXA1Ac2-26 peptide, a possible therapeutic approach in inflammatory ocular diseases. Gene 614: 26-36, 2017.
42. Stuqui B, de Paula-Silva M, Carlos CP, Ullah A, Arni RK, Gil CD and Oliani SM: Ac2-26 mimetic peptide of annexin A1 inhibits local and systemic inflammatory processes induced by bothrops moojeni venom and the Lys-49 phospholipase A2 in a rat model. PLoS One 10: e0130803, 2015.

43. Solito E, de Coupade C, Parente L, Flower RJ and Russo-Marie F: IL-6 stimulates annexin 1 expression and translocation and suggests a new biological role as class II acute phase protein. Cytokine 10: 514-521, 1998

44. Perucci LO, Carneiro FS, Ferreira CN, Sugimoto MA, Soriani FM, Martins GG, Lima KM, Guimarães FL, Teixeira AL and Dusse LM: Annexin A1 is increased in the plasma of preeclamptic women. PLoS One 10: e0138475, 2015.

45. Perucci LO, Vieira ELM, Teixeira AL, Gomes KB, Dusse LM and Sousa LP: Decreased plasma concentrations of brain-derived neurotrophic factor in preeclampsia. Clin Chim Acta 464: 142-147, 2017.

46. Zhao Y, Li X, Gong J, Li L, Chen L, Zheng L, Chen Z, Shi J and Zhang H: Annexin A1 nuclear translocation induces retinal ganglion cell apoptosis after ischemia-reperfusion injury through the p65/IL-1beta pathway. Biochim Biophys Acta 1863: 1350-1358, 2017.

47. Mor G and Abrahams VM: Potential role of macrophages as immunoregulators of pregnancy. Reprod Biol Endocrinol 1: 119, 2003.

48. Belo L, Caslake M, Gaffney D, Santos-Silva A, Pereira-Leite L, Quintanilha A and Rebelo I: Changes in LDL size and HDL concentration in normal and preeclamptic pregnancies. Atherosclerosis 162: 425-432, 2002.

49. Zou Y, Zuo Q, Huang S, Yu X, Jiang Z, Zou S, Fan M and Sun L: Resveratrol inhibits trophoblast apoptosis through oxidative stress in preeclampsia-model rats. Molecules 19: 20570-20579, 2014.

50. Huang L, Liao L, Wan Y, Cheng A, Li M, Chen S, Li M, Tan X and Zeng G: Downregulation of Annexin A1 is correlated with radioresistance in nasopharyngeal carcinoma. Oncol Lett 12: 5229-5234, 2016.

51. D'Acunto CW, Fontanella B, Rodriquez M, Taddei M, Parente L and Petrella A: Histone deacetylase inhibitor FR 235222 sensitizes human prostate adenocarcinoma cells to apoptosis through up-regulation of Annexin A1. Cancer Lett 295: 85-91, 2010.

52. Vago JP, Nogueira CR, Tavares LP, Soriani FM, Lopes F, Russo RC, Pinho V, Teixeira MM and Sousa LP: Annexin A1 modulates natural and glucocorticoid-induced resolution of inflammation by enhancing neutrophil apoptosis. J Leukoc Biol 92: 249-258, 2012.

53. Bao L, Devi YS, Bowen-Shauver J, Ferguson-Gottschall S, Robb L and Gibori G: The role of interleukin-11 in pregnancy involves up-regulation of alpha2-macroglobulin gene through janus kinase 2-signal transducer and activator of transcription 3 pathway in the decidua. Mol Endocrinol 20: 3240-3250, 2006.

This work is licensed under a Creative Commons Attribution-NonCommercial-NoDerivatives 4.0 International (CC BY-NC-ND 4.0) License. 